\section{5-3.4 DISEASE BURDENS ATTRIBUTABLE TO HIGHER THAN OPTIMAL SODIUM INTAKES: CHALLENGES IN ESTIMATING EXPOSURES AND EFFECT SIZES}

doi:10.1136/jech.2011.142976b.52

J Powles, ${ }^{*}$ S Fahimi. Department of Public Health and Primary Care, University of Cambridge, Cambridge, UK

Introduction Global burdens of cardiovascular disease are increasing as populations age. Salt contributes to vascular risk by increasing blood pressure but there have been no reported estimates of its contribution to global and regional disease burdens.

Methods Using a comparative risk assessment framework we identified effects of salt on intermediate (systolic blood pressure (SBP)) and disease (stomach cancer (SC)) outcomes that met the WHO criteria for causality at the "probable" or "convincing" level. Effect sizes were taken from meta-analyses of cohort studies (SC, risk related to intakes) and of randomised controlled trials of sodium reduction (SBP, effect related to urinary sodium excretion). Effect modification by age (for SBP) was estimated by re-analysis of a meta-analysis data set. Relative effects in populations of African ancestry came from 2 randomised controlled trials.

Regional age and sex specific exposure levels were assessed from published and unpublished reports of $24 \mathrm{~h}$ urinary excretion (assumed to be $90 \%$ of intake). Work on methods for extending exposure estimates using dietary survey data are on-going. These inputs, together with regional SC incidence and SBP distributions will allow computation of attributable disease burdens.

Results For study years 1990 (2005) estimates of urinary sodium excretion were available for 36 (22) countries from 16 (13) GBD regions. Only 3 (4) were from nationally representative samples. Work on attributable burdens is on-going and will be presented.
Conclusion Estimating disease burdens attributable to higher than optimal salt intake will prove feasible. Uncertainty will be increased by the limitations of the available exposure data.

\section{5-3.5 REVISITING THE CARDIOVASCULAR RISK TRANSITION: THE ASSOCIATIONS OF METABOLIC RISK FACTORS WITH NATIONAL INCOME SINCE BETWEEN 1980 AND 2008}

doi:10.1136/jech.2011.142976b.53

M Ezzati.* Imperial College London, London, UK

Introduction Cardiovascular diseases and their metabolic risk factors including overweight and obesity, blood pressure, cholesterol, and glucose have been predicted to rise with increasing income, originally referred to as the "diseases of affluence" paradigm. Our aim was to examine the associations between metabolic risk factors and national income since 1980 .

Methods We used estimates of age-standardised mean body mass index (BMI), systolic blood pressure (SBP), serum total cholesterol (TC) and fasting plasma glucose from a pooled analysis of population-based data. We assesses associations between risk factor levels and national income, measured as annual per-capita GDP converted to international dollars in 1990 and urbanisation, as proportion of a country's population that lived in urban areas in 1980, 1990, 2000, and 2008 .

Results In 1980, all four risk factors were associated with national income, with the association stronger for TC and BMI, and weaker for SBP and fasting plasma glucose. In 2008, TC was the only risk factor with strong association. BMI was highest in mid-income countries and SBP had an inverse association.

Conclusions The burden of metabolic risk factors has increasingly shifted to low- and middle-income countries, especially for SBP. 